\title{
Estrutura de capital e desempenho: evidência das companhias abertas de construção e engenharia listadas na BM\&FBovespa
}

\author{
Josimar Pires da Silva \\ Universidade de Brasilia \\ josimarnx@yahoo.com.br \\ Raiara Pinheiro Ferreira \\ Universidade Paulista \\ raiarafer@gmail.com \\ Tatiane Segalla Zappani \\ Universidade do Estado de Mato Grosso \\ tatiane_segalla@live.com \\ Rafael Martins Noriller \\ Universidade de Brasilia \\ rafael.mnoriller@gmail.com
}

\begin{abstract}
RESUMO
O presente trabalho objetiva analisar a relação entre estrutura de capital e desempenho das empresas dos subsetores de Construção civil e Construção e engenharia listadas na BM\&FBovespa. A amostra é composta por 32 empresas durante os anos-calendário de 2009 e 2015, resultando em 224 observações. Para efeitos de análise dos dados, foi considerado o ROA como proxy para o desempenho. Os resultados da pesquisa corroboram a relação negativa entre a estrutura de capital e o desempenho, uma vez que quanto maior utilização de capital de terceiros, menor o retorno. No geral os resultados convergiram com Chadha e Sharma (2015), Lara e Mesquita (2008) e Pamplona, Magro e Silva (2015) e contrariam os resultados de Machado et al (2015).
\end{abstract}

Palavras-chave: Engenharia. Construção Civil. Desempenho. Estrutura de Capital.

\begin{abstract}
The present work aims to analyze the relationship between capital structure and performance of the companies of the Civil Construction and Construction and engineering subsectors listed on BM\&FBovespa. The sample is composed of 32 companies during the calendar years of 2009 and 2015, resulting in 224 observations. For the purposes of data analysis, ROA was considered as a proxy for performance. The results of the research corroborate the negative relationship between capital structure and performance, since the higher the use of third-party capital, the lower the return. Overall the results converged with Chadha and Sharma (2015), Lara and Mesquita (2008) and Pamplona, Magro and Silva (2015) and its contradicts Machado et al (2015).
\end{abstract}

Keywords: Engineering. Construction. Performance. Capital structure. 


\section{INTRODUÇÃO}

A decisão de estrutura de capital é uma das decisões mais importantes tomadas por qualquer empresa (e.g. DANG; KIM; SHIN, 2014; CHADHA; SHARMA, 2015). Uma das questões importantes durante a tomada de decisão de estrutura de capital é lidar com a determinação da estrutura de capital ótima (CHADHA; SHARMA, 2015; SALIM; YADAV, 2012) uma vez que a administração tem que defini-la de forma a maximizar o valor da firma e continuidade.

No entanto, as empresas têm um nível diferente de alavancagem e os gestores tentam alcançar o melhor conjunto para atingir uma estrutura de capital ideal (SALIM; YADAV, 2012). Por sua vez, Leland e Toft (1996) expõem que fatores como incentivos fiscais, i.e., juros oriundos das dívidas, auxiliam na tomada de decisão por parte das empresas.

A estrutura de capital consiste em dívida, ações ordinárias e ações preferenciais que são usadas para financiar os projetos da empresa. A empresa aumenta o capital próprio por meio da emissão de ações ordinárias e preferenciais, enquanto o capital de terceiros pode ser elevado na forma de empréstimos a recolher, notas a recolher, obrigações, debêntures e outros (CHADHA; SHARMA, 2015).

Modigliani e Miller (1958) sugerem que em um mercado de capitais perfeito, as estratégias não afetam o valor da empresa, mas depois argumentam que o valor da empresa pode ser aumentado mudando a estrutura de capital devido à vantagem fiscal da dívida. No entanto, estas suposições não se mantêm no mundo real. A literatura sugere que existe uma estrutura de capital ótima, mas não existe uma metodologia específica para garantir que eles alcancem um nível ótimo de dívida. No entanto, a teoria financeira fornece alguma ajuda para entender como o conjunto de financiamento escolhido afeta o valor da empresa (SALIM; YADAV, 2012).

A literatura prévia tem destacado uma ampla gama de mecanismos relativos a estrutura de capital, principalmente nas pesquisas em nível internacional (BERGER; PATTI, 2006; CHADHA; SHARMA, 2015; CHEUNG et al., 2013; JO; KIM; PARK, 2014; LAUTERBACH; VANINSKY, 1999; MARGARITIS; PSILLAKI, 2010; PHUNG; MISHRA, 2015; SALIM; YADAV, 2012; WAHBA, 2014), e, algumas delas relacionadas ao desempenho empresarial, e.g., Perresim, Santos e Lucente (2014) no setor bancário brasileiro e Lara e Mesquita (2008) sem segregação setorial.

Em consonância ao trabalho de Modigliani e Miller (1958), Machado et al (2015) atentam para ausência de que a estrutura de capital está diretamente relacionada com o 
desempenho das empresas, especificamente brasileiras listadas na BM\&FBovespa. Em âmbito setorial, Gomes e Santos (2015), no setor petrolífero brasileiro, destacam que empresas com maior endividamento conseguem maior retornos superiores aos acionistas e considerando empresas familiares Pamplona, Magro e Silva (2015) encontraram relação positiva (negativa) entre o endividamento e o desempenho econômico no Brasil (Portugal).

Neste contexto, o trabalho de Leland e Toft (1996) destaca que o aumento de dívida por parte da empresa aumenta a percepção de risco por parte do investidor reportando a substituição por ativos com menor risco. Já DeAngelo e Stulz (2015) corroboram afirmando que a estrutura de capital acaba interferindo na percepção de risco por parte dos investidores, especificamente no setor financeiro.

Um dos setores que tem apresentado destacado desempenho, no contexto brasileiro, é o setor de construção civil e engenharia, principal empregador de mão de obra dos últimos anos, gerando milhares de vagas de empregos, devido ao crescimento do setor, da facilidade de acesso ao crédito e da expansão industrial em nível nacional (COSTA, 2003). No entanto, as pesquisas recentes não demonstram qual melhor forma de capital para esse grupo de empresas.

O International Monetary Fund (IMF) (2016) complementa destacando a importância do setor de construção civil para economia brasileira que desde o início da recessão econômica enfrenta destruição de empregos formais, justificado pelas dificuldades de captação de financiamentos e cenário político/econômico. O Setor responde, ainda, por aproximadamente 300 bilhões de reais em valor adicionado ao Produto Interno Bruto (PIB) brasileiro para os anos-calendário de 2014, 2015 e 2016 (BRASIL, 2017).

Desse modo, destaca-se como problema de pesquisa a ser respondido: Qual a relação entre o desempenho e a estrutura de capital das empresas brasileiras de capital aberto listadas nos subsetores de Construção civil e Construção e engenharia da BM\&FBovespa? A fim de responder a esse questionamento, esta investigação tem como objetivo analisar a relação entre estrutura de capital e desempenho das empresas dos subsetores de Construção civil e Construção e engenharia listadas na BM\&FBovespa.

Até a realização da pesquisa, não foram encontradas pesquisas no contexto brasileiro que se propusessem a investigar a relação entre estrutura de capital e desempenho das empresas dos subsetores de Construção civil e Construção e engenharia. Pesquisas relativas aos subsetores se fazem tempestivas, sobretudo, em virtude do crescimento do setor observado nos últimos anos. Poucas pesquisas têm dedicado atenção necessária em países em desenvolvimento, como é o caso do Brasil. Outra motivação diz respeito a ausência de pesquisas 
relativas a esses subsetores, mas que, tem elevado destaque, sobretudo pela geração de empregos diretos e indiretos, tanto nas grandes empresas, quantos nas pequenas e médias.

\section{PLATAFORMA TEÓRICA E DESENVOLVIMENTO DA HIPÓTESE}

Sempre se acredita que a determinação da estrutura de capital ótima é uma questão desconcertante nas finanças corporativas (WAHBA, 2014). O tema subjacente é que a capacidade da empresa de explorar uma estrutura de capital apropriada provavelmente resultará em uma vantagem competitiva sustentável (BARTON; GORDON, 1988). Para verificar essa premissa, os pesquisadores têm procurado investigar a associação entre várias características nos níveis de indústria (KESTER, 1986; MARSH, 1982; MICHAELAS; CHITTENDEN; POUTZIOURIS, 1999; TITMAN; WESSELS, 1988) e vários arranjos de estrutura de capital.

Evidências empíricas mostram que as características específicas da empresa ou microfatores desempenham um papel significativo na determinação da estrutura de capital de uma empresa (CHADHA; SHARMA, 2015). Donaldson (1985) argumentou que a dependência da dívida ou da equidade muda à medida que a propriedade da empresa muda e resulta em mudanças nas metas financeiras da empresa.

O desempenho da empresa é significativamente afetado por vários fatores e a estrutura de capital é um dos fatores significativos entre eles. Muitos estudos empíricos têm sido feitos para explorar se há alguma relação (positiva, negativa ou sem relação) entre o desempenho da empresa e a estrutura de capital e esses estudos produziram resultados mistos (SALIM; YADAV, 2012; PAMPLONA; MAGRO; SILVA, 2015).

Neste contexto, uma corrente de pesquisa se concentrou em examinar a relação entre a política da dívida e o desempenho das empresas. O aumento do nível médio de endividamento entre as empresas e o papel esperado que o nível da dívida pode desempenhar não apenas em interesses convergentes (ou divergentes) entre gestores e investidores, mas também na maximização da riqueza dos acionistas são exemplos das razões que são frequentemente apresentados na literatura para justificar a importância de estudar essa relação (WAHBA, 2014).

Da mesma forma, estudos empíricos que examinaram a relação entre a dívida e o desempenho das empresas chegam a conclusões concorrentes. Enquanto alguns estudos (ABOR, 2005; BERGER; PATTI, 2006; GHOSH; NAG; SIRMANS, 2000; HADLOCK; JAMES, 2002; RODEN; LEWELLEN, 1995; TAUB, 1975) apoiaram a correlação positiva entre a política da dívida e o desempenho da empresa, outros estudos (BOOTH et al., 2001; CHAKRABORTY, 2010; CHINAEMEREM; ANTHONY, 2012; FAMA; FRENCH, 1998; 
FRIEND; LANG, 1988; GLEASON; MATHUR; MATHUR, 2000; HUANG; SONG, 2006; KESTER, 1986; MAJUMDAR; CHHIBBER，1999; MARGARITIS; PSILLAKI，2010; PRATHEEPKANTH, 2011; RAJAN; ZINGALES, 1995; SALEHI; BIGLAR, 2009; SALIM; YADAV, 2012; SIMERLY; LI, 2000; SINGH; FAIRCLOTH, 2005; SPIESS; AFFLECKGRAVES, 1999; TITMAN; WESSELS, 1988; WALD, 1999; WIWATTANAKANTANG, 1999) argumentaram que a dívida tende a um desempenho inferior da empresa. No entanto, outros estudos (EBAID, 2009; KHAN, 2012; PHILLIPS; SIPAHIOGLU, 2004; SAN; HENG, 2011) concluem que a dívida não tem impacto no desempenho das empresas.

Roden e Lewellen (1995) examinam a estrutura de capital de 48 firmas americanas durante o período de 1981-1990 e revelaram uma relação positiva entre rentabilidade e estrutura de capital. Resultados semelhantes foram documentados por Ghosh, Nag e Sirmans (2000). Hadlock e James (2002) sugerem corporações com alto nível de rentabilidade usam alto nível de dívidas. Abor (2005) relata uma relação positiva entre a estrutura de capital, medida por dívida de curto prazo e dívida total, e o desempenho no período 1998-2002 nas empresas ganianas.

Salim e Yadav (2012) descobriram que há uma correlação negativa entre Retorno sobre Ativo (ROA), Retorno sobre Patrimônio Líquido (ROE) e alavancagem financeira, mas uma correlação positiva entre Q de Tobin e alavancagem financeira. Chinaemerem e Anthony (2012) encontraram uma relação negativa entre ROA, ROE e alavancagem financeira.

Salehi e Biglar (2009) argumentam que há uma relação inversa entre ROE, ROA e alavancagem. Kester (1986) encontrou uma relação negativa entre estrutura de capital e desempenho (lucratividade) nos EUA e no Japão. Resultados semelhantes foram relatados por Friend e Lang (1988), Titman e Wessels (1988) das empresas norte-americanas, Rajan e Zingales (1995) nos países do G-7, Wald (1999) nos países desenvolvidos. Além disso, Wewattanakantang (1999) relatou uma relação negativa entre alavancagem do patrimônio líquido e do valor de mercado e ROA de 270 empresas tailandesas.

Também encontraram uma correlação negativa entre alavancagem e desempenho (lucros antes de juros e impostos dividido pelos ativos totais) (HUANG; SONG, 2006). Chakraborty (2010) empregou duas medidas de desempenho, incluindo provisão de lucro antes de juros, impostos e depreciação para ativos totais e relação de fluxos de caixa para ativos totais e duas medidas de alavancagem, incluindo provisão do total de empréstimos para ativos e de passivo e capital próprio e relataram uma relação negativa entre estes. 
Pratheepkanth (2011) analisou a estrutura de capital e seu impacto sobre a capacidade de desempenho financeiro durante 2005 a 2009 de empresas de negócios no Sri Lanka. Os resultados mostrados a relação entre a estrutura de capital e o desempenho financeiro é negativo.

Por outro lado, Ebaid (2009) argumentou que a estrutura de capital não tem impacto nos parâmetros de desempenho financeiro da empresa como ROA e ROE. Os resultados da pesquisa de Khan (2012) foram consistentes com o modelo de custo da agência de Jensen e Mecklin (1976) e não encontraram nenhum impacto significativo da eficiência na alavancagem. Há evidências de não-linearidades na relação entre o tipo de propriedade com estrutura de capital e o desempenho das empresas.

Ebaid (2009) investigou o impacto da escolha da estrutura de capital no desempenho de 64 empresas entre 1997 e 2005 no mercado de capitais egípcio. Ele emprega três medidas contábeis, incluindo ROA, ROE e margem bruta de lucro, e conclui as escolhas de estrutura de capital, em geral, tem um impacto fraco sobre o desempenho da empresa. San e Heng (2011) em sua pesquisa focada em empresas de construção que estão listados na Malásia de 20052008, o resultado mostra que há uma relação entre a estrutura de capital e o desempenho corporativo e há evidências que mostram que não relação entre as variáveis foram investigadas.

Em síntese, a evidência empírica fornece sugestões variadas e inconsistentes sobre a relação entre a alavancagem financeira e o desempenho da empresa em Economia desenvolvida (CHADHA; SHARMA, 2015) e em desenvolvimento. Além disso, existem estudos muito limitados que empiricamente testar esta relação em países em desenvolvimento como o Brasil. $\mathrm{O}$ presente estudo reforça a literatura sobre o efeito da alavancagem financeira sobre $\mathrm{o}$ desempenho das empresas, testando empiricamente a relação entre alavancagem financeira e desempenho da empresa no setor da construção civil e engenharia.

Nesse contexto, a discussão anterior indica que, embora a dívida e a propriedade gerencial, quando usadas na separação, possam ser boas ou ruins para o desempenho da empresa, sua interação pode ter consequências diferentes no desempenho da empresa. A maioria das pesquisas apontam uma relação negativa entre ROA e estrutura de capital. Em linhas com esses argumentos, a hipótese da pesquisa é:

H1: Existe uma relação significante e negativa entre a estrutura de capital (capital de terceiros/capital próprio) e o desempenho das companhias de construção e engenharia listadas na BM\&FBovespa, ceterius paribus. 


\section{METODOLOGIA}

Esta pesquisa é caracterizada como descritiva com abordagem quantitativa. Foram utilizadas informações dos demonstrativos financeiros empresariais, coletados a partir da base de dados no sítio eletrônico da BM\&FBovespa. A amostra inicial foi composta por 18 empresas do subsetor de construção civil e 16 empresas do subsetor construção e engenharia, totalizando 34. Foram excluídas da amostra duas empresas que possuíam dados para todo o período da amostra, totalizando 32 empresas.

No que tange ao período analisado, a pesquisa consiste nos anos-calendário de 2009 e 2015, compreendendo um período de 7 anos consecutivos, resultando em 224 observações. Períodos anteriores não foram considerados pelo fato da ausência de convergência total às Normas Internacionais de Contabilidade (full IFRS) e também, de acordo com o IMF (2017), o período mais difícil da crise financeira mundial 2007 e 2008, com alta volatilidade no mercado acionário brasileiro.

No que tange a proxy de desempenho, a presente pesquisa toma por base os trabalhos de Chadha e Sharma (2015), Wahba (2014), Salim e Yadav (2012) e Margaritis e Psillaki (2010) e foi utilizado o ROA, frequentemente abordado na literatura. A base para mensuração do ROA é o lucro operacional, conforme equação 1 .

$$
R O A=\frac{\text { Lucro Operacional }}{\text { Ativos }}
$$

Foi utilizada como variável dependente, relativa ao desempenho, ROA, e, como variável independente a estrutura de capital (EC), obtidos conforme equação 1 e 2.

Dessa forma, o ROA é usado nesse estudo como proxy para desempenho, e, utilizado como variável dependente na regressão OLS - equação (2) - para testar a associação entre estrutura de capital e desempenho, conforme modelo com dados em painel, adaptado a partir das pesquisas de Chadha e Sharma (2015), Wahba (2014), Salim e Yadav (2012) e Margaritis e Psillaki (2010), a seguir:

$$
R O A_{i, t}=\beta_{0}+\beta_{1} E C_{i, t}+\beta_{2} R P_{i, t}+\beta_{3} R T_{i, t}+\beta_{4} I M O_{i, t}+\beta_{5} P D R_{i, t}+\beta_{6} G R O W T H_{i, t}+\varepsilon_{i, t}
$$

Em que:

$R O A=$ Lucro operacional dividido pelo ativo.

$E C=$ Passivo dividido pelo patrimônio líquido

$R P=$ Remuneração do Capital Próprio.

$R T=$ Remuneração do Capital de Terceiros. 
$I M O=$ Proporção dos Ativos Imobilizados em relação aos Ativos totais.

$P D R=$ Proporção de despesas operacionais em relação as receitas operacionais.

GROWTH $=$ Crescimento das Receitas.

As variáveis de controle foram inseridas no modelo com objetivo de buscar maior robustez aos resultados encontrados. As variáveis e suas mensurações são resumidas e justificadas no quadro 1 .

Quadro 1 - Resumo das variáveis explanatórias

\begin{tabular}{|c|c|c|}
\hline $\begin{array}{c}\text { Variáveis } \\
\text { Explanatórias }\end{array}$ & $\begin{array}{c}\text { Sinal } \\
\text { Esperado }\end{array}$ & Justificativa \\
\hline $\begin{array}{l}\text { Estrutura de Capital } \\
\text { (EC) }\end{array}$ & Negativo & $\begin{array}{l}\text { A estrutura de capital tem impacto negativo no desempenho da } \\
\text { empresa medido pelo ROA (SALIM; YADAV, 2012). O } \\
\text { desempenho da empresa está relacionado negativamente à } \\
\text { estrutura de capital (ABOR, 2005; RAJAN; ZINGALES, 1995; } \\
\text { PAMPLONA; MAGRO; SILVA, 2015). }\end{array}$ \\
\hline $\begin{array}{l}\text { Remuneração de } \\
\text { Capital Próprio (RP) }\end{array}$ & Positivo & $\begin{array}{l}\text { Quanto maior a remuneração de capital próprio, maiores } \\
\text { possibilidades de investimentos dos detentores de ações. }\end{array}$ \\
\hline $\begin{array}{l}\text { Remuneração } \text { de } \\
\text { Capital de Terceiros } \\
\text { (RT) }\end{array}$ & Negativo & $\begin{array}{l}\text { Quanto maior a remuneração de capitais de terceiros, menores } \\
\text { lucros, e, portanto, menor desempenho. }\end{array}$ \\
\hline Imobilização (IMO) & Negativo & $\begin{array}{l}\text { A tangibilidade dos ativos tem uma correlação negativa com o } \\
\text { ROA, que representa menos retorno devido ao elevado custo da } \\
\text { dívida (CHADHA; SHARMA, 2015). }\end{array}$ \\
\hline $\begin{array}{l}\text { Relação } \\
\text { despesas/receitas } \\
\text { operacionais (PDR) }\end{array}$ & Negativo & $\begin{array}{l}\text { A eficiência operacional tem um impacto positivo no } \\
\text { desempenho das empresas. Quanto maior eficiência medida pela } \\
\text { relação entre despesas e receitas operacionais, maior o } \\
\text { desempenho. }\end{array}$ \\
\hline $\begin{array}{ll}\text { Crescimento } & \text { das } \\
\text { Receitas (Growth) } & \end{array}$ & Positivo & $\begin{array}{l}\text { O crescimento das vendas está positivamente correlacionado } \\
\text { com o ROA. As empresas com altas vendas resultam em maior } \\
\text { rentabilidade. (CHADHA; SHARMA, 2015). }\end{array}$ \\
\hline
\end{tabular}

Fonte: Elaborado pelos autores.

Como limitação principal a pesquisa foi realizada utilizando um setor especifico como amostra - construção civil e engenharia - e os resultados podem ser inferidos para tal setor, de forma que não podem ser generalizadas para os demais setores atingindo a totalidade das empresas brasileiras. Quanto a proxy para a qualidade desempenho, foi utilizado o ROA. Desta maneira, a presente investigação se limita a demonstrar a relação entre estrutura de capital e desempenho com base no ROA, não podendo fazer maiores inferências quanto a outras medidas de desempenho. 


\section{RESULTADOS E DISCUSSÃO}

Com o intuito de verificar a relação entre estrutura de capital e desempenho, foi utilizada como proxy para o desempenho da empresa, o ROA (lucro operacional da empresa dividido pelos investimentos.

A tabela 1 evidencia a estatística descritiva das demais variáveis analisadas, nos quais os resultados demonstram que: a) a maioria das empresas utilizam maior proporção de capital de terceiros em relação ao capital próprio, na estrutura de financiamento; b) a remuneração dos capitais de terceiros e próprio são em média equivalentes, cerca de 3\%; c) as despesas operacionais representam na média, cerca de $25 \%$ das receitas; e; d) o crescimento das receitas, em média, representa $15 \%$ ao período.

Tabela 1: Estatística descritiva

\begin{tabular}{cccccc}
\hline Variável & Média & Mediana & Máximo & Mínimo & Desvio Padrão \\
\hline \hline ROA & 0,045846 & 0,054406 & 0,459414 & $-0,438192$ & 0,108819 \\
EC & 2,412787 & 1,429748 & 31,56887 & $-8,075945$ & 4,055129 \\
RP & 0,036936 & 0,044236 & 0,542520 & $-0,443541$ & 0,098485 \\
RT & 0,053316 & 0,038043 & 0,537173 & 0,000000 & 0,062921 \\
GROWTH & 0,096125 & 0,063262 & 1,000000 & $-10,9429$ & 1,016005 \\
PDR & 0,250353 & 0,149321 & 2,078202 & $-0,343501$ & 0,361447 \\
IMO & 0,110826 & 0,016552 & 1,000000 & 0,000145 & 0,179149 \\
\hline
\end{tabular}

Fonte: elaborada pelos autores.

A Tabela 2 apresenta os coeficientes de correlação dos pares de variáveis. Os testes (não relatado) mostram que, não há nenhuma variável de previsão que produz um fator de inflação da variância maior ou igual a 10, corroborando que multicolinearidade não é um problema para a estimativa da equação de regressão (2). A relação de maior valor foi entre as variáveis EC e RCP (-0,328111), RCP e GROWTH $(0,254056)$ e EC e GROWTH $(-0,215138)$, indicando baixa correlação entre as variáveis do modelo (explanatórias).

Além disso, também foram analisados os pressupostos básicos da regressão OLS: normalidade, homocedasticidade e autocorrelação dos resíduos, através dos testes de JarqueBera, teste de White e de Breusch-Pagan-Godfrey e de Durbin-Watson, respectivamente. Os testes indicaram que os resíduos seguem uma distribuição normal, não há autocorrelação entre os resíduos e os mesmos são homocedásticos, o que atende aos pressupostos da regressão linear. 
Tabela 2 - Análise da correlação das variáveis

\begin{tabular}{cccccccc}
\hline & ROA & EC & RCP & RCT & GROWTH & PDR & IMO \\
\hline \hline ROA & 1 & & & & & & \\
EC & $-0,405959$ & 1 & & & & & \\
RCP & 0,951844 & $-0,328111$ & 1 & & & & \\
RCT & $-0,169539$ & 0,030645 & $-0,199080$ & 1 & & & \\
GROWTH & 0,240333 & $-0,215138$ & 0,254056 & $-0,002225$ & 1 & & \\
PDR & 0,107366 & $-0,006777$ & 0,076206 & $-0,017614$ & $-0,021450$ & 1 & \\
IMO & $-0,110328$ & 0,033993 & $-0,104614$ & 0,192383 & 0,015638 & $-0,002412$ & 1 \\
\hline
\end{tabular}

Fonte: elaborada pelos autores.

Suplementarmente foram analisados os demais pressupostos básicos, isto é, normalidade dos resíduos, homocedasticidade e autocorrelação. No que tange a normalidade dos resíduos, foi realizado o teste de Jarque-Bera, o qual indicou que os resíduos não seguem uma distribuição normal. No entanto utilizou-se como suporte, o Teorema do Limite Central, com base em Gujarati e Porter (2011), em que para amostras maiores que 100 observações, presume-se a distribuição normal, i.e., o pressuposto da normalidade está restrito para as amostras que contêm menos de 100 observações.

Quanto a autocorrelação dos resíduos, utilizou-se o teste de Durbin-Watson o qual obteve-se o valor 2,0283, demonstrando a não existência de autocorrelação dos resíduos. Para o pressuposto da homocedasticidade dos resíduos, utilizou-se o teste de Breusch-PaganGodfrey, o qual demonstrou a existência de heterocedasticidade, a qual foi corrigida por meio do método de covariância de White.

Adicionalmente foram realizados os testes de Breusch-Pagan-Godfrey, Hausman e F (Chow) para detectar o melhor modelo e os resultados evidenciaram a existência do efeito grupo. Dessa forma, foi realizada regressão com efeitos fixos para confirmar os sinais esperados das variáveis e os resultados podem ser visualizados na tabela 3.

As colunas A, B, C e D da tabela 3, revelam os resultados da regressão OLS estimada da equação (2) em que foram estimados da seguinte forma: coluna A, sem variável de controle; colunas B, C e D com a inserção das variáveis de controle (RCP, RCT, GROWTH, PDR e IMO). De forma adicional, a coluna E, mostra o resultado com a inserção da variável ROE como variável dependente. 
Tabela 3 - Análise da regressão das variáveis

\begin{tabular}{|c|c|c|c|c|c|}
\hline VARIÁVEIS & $\begin{array}{c}\mathrm{A} \\
\text {-value } \\
\text { (sig) }\end{array}$ & 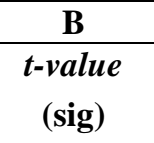 & 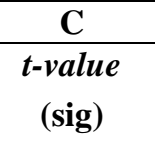 & $\begin{array}{c}\mathrm{D} \\
\text {-value } \\
\text { (sig) }\end{array}$ & $\begin{array}{c}\mathrm{E} \\
t \text {-value } \\
\text { (sig) }\end{array}$ \\
\hline \multirow[t]{2}{*}{ INTERCEPTO } & 6,6885 & 7,9474 & 8,6818 & 8,7030 & 1,627182 \\
\hline & 0,0000 & 0,0000 & 0,0000 & 0,0000 & 0,1058 \\
\hline \multirow[t]{2}{*}{$\mathrm{EC}$} & $-1,9458$ & $-3,0072$ & $-3,4294$ & $-3,0712$ & $-3,856704$ \\
\hline & 0,0532 & 0,0031 & 0,0008 & 0,0025 & 0,0002 \\
\hline \multirow[t]{2}{*}{$\mathrm{RCP}$} & & 36,8896 & 39,3455 & 39,1065 & 17,91914 \\
\hline & & 0,0000 & 0,0000 & 0,0000 & 0,0000 \\
\hline \multirow[t]{2}{*}{$\mathrm{RCT}$} & & $-1,2897$ & $-1,4992$ & $-1,4949$ & 1,803888 \\
\hline & & 0,1991 & 0,1359 & 0,1371 & 0,0733 \\
\hline \multirow[t]{2}{*}{ GROWTH } & & 1,5826 & 1,3485 & 1,3452 & 2,276172 \\
\hline & & 0,1156 & 0,1795 & 0,1806 & 0,0243 \\
\hline \multirow[t]{2}{*}{ PDR } & & & $-19,8272$ & $-19,7598$ & $-0,212977$ \\
\hline & & & 0,0000 & 0,0000 & 0,8316 \\
\hline \multirow[t]{2}{*}{ IMO } & & & & $-0,0287$ & $-1,297162$ \\
\hline & & & & 0,9771 & 0,1966 \\
\hline $\mathbf{R}^{2}$ & $\mathbf{0 , 7 1 3 4}$ & 0,9864 & 0,9864 & 0,9864 & 0,817874 \\
\hline $\mathbf{R}^{2}$ ajustado & 0,6644 & 0,9864 & 0,9830 & 0,9829 & 0,772950 \\
\hline $\mathbf{F}$ & 14,5497 & 305,5567 & 294,3170 & 284,6258 & 18,20555 \\
\hline (sig) & $\mathbf{0 , 0 0 0 0}$ & $\mathbf{0 , 0 0 0 0}$ & $\mathbf{0 , 0 0 0 0}$ & $\mathbf{0 , 0 0 0 0}$ & $\mathbf{0 , 0 0 0 0}$ \\
\hline
\end{tabular}

Variável Dependente: Desempenho da Empresa (ROA). Variáveis independentes: Estrutura de Capital (EC); Remuneração do Capital Próprio (RP); Remuneração do Capital de Terceiros (RT); Proporção dos ativos imobilizados em relação aos investimentos totais (IMO); Proporção de despesas operacionais em relação as receitas (PDR); e, Crescimento das receitas (Growth).

Fonte: dados da pesquisa.

Os dados da regressão (coluna A, tabela 3) evidenciaram que a variável $E C$ apresentou um coeficiente significante a 5\% ( $p$-valor $=0,0532)$ e negativo para com a variável dependente, especificamente ROA, corroborando assim a hipótese de que quanto maior a proporção de capitais de terceiros em relação ao capital próprio, das empresas nos subsetores de Construção civil e Construção e engenharia, menor desempenho. Desse modo, a hipótese de pesquisa foi corroborada indicando que há uma relação significante e positiva entre a utilização de capital próprio e desempenho das companhias pesquisadas, em linha com Salim e Yadav (2012) e divergente de Chadha e Shaharma (2015) e Lara e Mesquita (2008).

Com a inserção das variáveis de controle, especificamente remuneração do capital próprio (RCP), remuneração do capital de terceiros (RCT) e crescimento das receitas (GROWTH), o sinal da estatística se manteve negativo, e, apresentou um coeficiente significante a $1 \%(p$-valor $=0,0031)$, além de elevar o $R^{2}$ e o $R^{2}$ ajustado. A RCP evidenciou sinal positivo em relação ao ROA, como esperado (e.g. GOMES; SANTOS, 2015), visto que maior remuneração destes capitais, podem atrair maiores investimentos. As variáveis RCT e 
GROWTH, apresentaram sinais esperados negativo e positivo, respectivamente, conforme esperado, no entanto, não apresentaram coeficientes significantes a 10\%. Quanto a variável GROWTH, o sinal esperado convergiu com o resultado de Chadha e Sharma (2015) em que o crescimento das vendas está positivamente correlacionado com o ROA e as empresas com altas vendas resultam em maior rentabilidade.

A coluna $\mathrm{C}$, mostra o resultado com a inserção da variável proporção das despesas operacionais em relação as receitas operacionais (PDR). Essa variável apresentou coeficiente negativo e significativo a $1 \%$ ( $p$-valor $=0,0000)$, e, não provocou modificações no comportamento das demais variáveis do modelo. O resultado do coeficiente demonstra que quanto maior a eficiência operacional, maior o desempenho da empresa.

No que tange a coluna $\mathrm{D}$ da tabela 3, evidencia o resultado com todas as variáveis, isto é, a inserção da variável proporção dos ativos imobilizados em relação aos ativos totais (IMO). Essa variável apresentou um coeficiente negativo, corroborando o pressuposto de que a tangibilidade dos ativos tem uma correlação negativa com o ROA, que representa menos retorno devido ao elevado custo da dívida, convergindo com Chadha e Sharma (2015). No entanto, o coeficiente não foi significante a $10 \%$ ( $p$-valor $=0,9771)$. Quanto as demais variáveis, não apresentaram modificações significativas, permanecendo os mesmos sinais, já obtidos. Especificamente em relação a EC, apresentou um coeficiente significante a $1 \%$ ( $p$ valor $=0,0025)$ e negativo para com a variável dependente (ROA).

De forma complementar, foi inserida variável retorno sobre patrimônio líquido (ROE) como variável dependente. Apenas os coeficientes das variáveis PDR e IMO não foram significativos. Quanto a sinal esperado, o comportamento da variável RCT (que era negativo) mudou para positivo, evidenciando que a remuneração do capital de terceiros impacta positivamente no ROE em contradição ao trabalho de Mesquita et al (2015). Esse fato pode ser justificado pelo benefício da dívida, uma vez que os juros pagos a terceiros, reduzem a base de cálculo do imposto de renda e da contribuição social, elevando o lucro.

Em comparação com os resultados previamente apresentados para o ROA, diferentemente do ROE, não era afetado pelo benefício da dívida uma vez para o cálculo daquele, utilizou-se o lucro operacional (mensurando a eficiência operacional da empresa). Afim de confirmar esse comportamento, foram estimados novos coeficientes, desta vez com o ROA calculado pela relação entre lucro líquido sobre ativos totais. Os resultados não tabulados apontaram significância para a variável RCT e sinal positivo, confirmando o pressuposto do 
benefício da dívida, e que, quanto maior a remuneração de capitais de terceiros, maior o desempenho, baseado na relação entre lucro líquido e ativos.

\section{CONSIDERAÇÕES FINAIS}

A proposta dessa pesquisa foi investigar a relação entre o desempenho e a estrutura de capital das empresas dos subsetores de Construção civil e Construção e engenharia listadas na BM\&FBovespa. Os resultados indicaram a estrutura de capital, medida pela relação entre capital de terceiros e capital próprio, impacta negativamente no desempenho das companhias abertas dos subsetores, uma vez que quanto maior proporção de capital próprio, maior desempenho. Uma possível justificativa é o elevado custo de capital de terceiros podendo reduzir o resultado dessas empresas.

Dessa maneira, foi possível confirmar a hipótese da pesquisa, a qual destaca que existe uma relação significante e negativa entre a estrutura de capital (capital de terceiros/capital próprio) e o desempenho das companhias listadas nos subsetores de Construção Civil e Construção e Engenharia listadas na BM\&FBovespa.

As variáveis RCP, RCT, GROWTH, PDR e IMO, apresentaram sinal esperado, uma vez que RCP e GROWTH, impactam positivamente no desempenho e RCT, PDR e IMO, impactam negativamente. No entanto, as variáveis RCT, GROWTH e IMO não apresentaram coeficientes significativos.

Em síntese, os resultados da pesquisa são correlatos com aqueles encontrados em pesquisas de nível nacional e internacional, relativas aos setores em geral. Dessa forma, o setor de construção civil e engenharia, no que tange a relação entre desempenho e estrutura de capital, segue o comportamento da média da indústria. Para futuras pesquisas poderiam ser considerados outros setores importantes para economia nacional, como comercial e de serviços.

\section{REFERÊNCIAS BIBLIOGRÁFICAS}

ABOR, J. The effect of capital structure on profitability: an empirical analysis of listed firms in Ghana. The Journal of Risk Finance, v. 6, n. 5, p. 438-445, 2005.

BARTON, S. L.; GORDON, P. J. Corporate Strategy and Capital Structure. Strategic Management Journal, v. 9, n. 6, p. 623-632, 1988.

BERGER, A. N. .; PATTI, E. B. DI. Capital structure and firm performance: A new approach to testing agency theory and an application to the banking industry. Journal of Banking and Finance, v. 30, n. 4, p. 1065-1102, 2006. 
BOOTH, L. et al. Capital Structures in Developing Countries. The Journal of Finance, v. 56, n. 1, p. 87-130, 2001.

BRASIL. PIB Brasil e Construção Civil. Disponível em:

http://www.cbicdados.com.br/menu/pib-e-investimento/pib-brasil-e-construcao-civil. Acesso em: 17 de julho de 2017.

CHADHA, S.; SHARMA, A. K. Capital Structure and Firm Performance: Empirical Evidence from India. The Journal of Business Perspective, v. 19, n. 4, p. 295-302, 2015.

CHAKRABORTY, I. Capital structure in an emerging stock market: The case of India.

Research in International Business and Finance, v. 24, n. 3, p. 295-314, 2010.

CHEUNG, Y. L. et al. Corporate Social Performance, Firm Valuation, and Industrial

Difference: Evidence from Hong Kong. Journal of Business Ethics, v. 114, n. 4, p. 625-631, 2013.

CHINAEMEREM, O. C.; ANTHONY, AND O. Impact Of Capital Structure On The Financial Performance Of Nigerian Firms. Arabian Journal of Business and Management Review, v. 1, n. 12, p. 43-61, 2012.

COSTA, Dayana Bastos. Diretrizes para concepção, implantação e uso de sistemas de indicadores de desempenho para empresas da construção civil. Mestrado em Engenharia Civil. UFRGS, 2003.

DA SILVEIRA, A. D. M.; BARROS, L. A. B. DE C. Determinantes da Qualidade da Governança Corporativa das Companhias Abertas Brasileiras. Journal of Chemical Information and Modeling, v. 14, n. 3, p. 160, 2008.

DANG, Viet Anh; KIM, Minjoo; SHIN, Yongcheol. Asymmetric adjustment toward optimal capital structure: Evidence from a crisis. International Review of Financial Analysis, v. 33, p. 226-242, 2014.

DEANGELO, Harry; STULZ, René M. Liquid-claim production, risk management, and bank capital structure: Why high leverage is optimal for banks. Journal of Financial Economics, v. 116, n. 2, p. 219-236, 2015.

DONALDSON, L. Organization design and the life cycles of products. Journal of Management Studies, v. 22, n. 1, p. 25-37, 1985.

EBAID, I. E.-S. The impact of capital-structure choice on firm performance: empirical evidence from Egypt. The Journal of Risk Finance, v. 10, n. 5, p. 477-487, 2009. FAMA, E. F.; FRENCH, K. R. Taxes, Financing Decisions, and Firm Value. The Journal of Finance, v. 53, n. 3, p. 819-843, 1998. 
INTERNATIONAL MONETARY FUND. Brazil - 2016 Article IV Consultation. 2016. Disponível em: https://www.imf.org/external/pubs/ft/scr/2016/cr16348.pdf. Acesso em: 17 de julho de 2017.

IMF's Response to the Global Economic Crisis. Disponível em:

http://www.imf.org/en/About/Factsheets/Sheets/2016/07/27/15/19/Response-to-the-GlobalEconomic-Crisis. Acesso em 07 de julho de 2017.

FRIEND, I.; LANG, L. H. P. An empirical test of the impact of self interest on corporate capital structure. The Journal of Finance, v. XLIII, n. 2, p. 271-282, 1988.

GHOSH, C.; NAG, R.; SIRMANS, C. F. The Pricing of Seasoned Equity Offerings: Evidence from REITs. Real Estate Economics, v. 28, p. 363-384, 2000.

GLEASON, K. C.; MATHUR, L. K.; MATHUR, I. The interrelationship between culture, capital structure, and performance: evidence from European retailers. Journal of Business Research, v. 50, n. 2, p. 185-191, 2000.

GOMES, Priscila Freitas de Souza; SANTOS, David Ferreira Lopes. Estrutura de Capital e Desempenho Financeiro do Setor Petrolífero Brasileiro. Revista de Ciências Jurídicas e Empresariais, v. 15, n. 1, 2015.

GUJARATI, D. N.; PORTER, D. C. Econometria Básica. [s.l: s.n.].

HADLOCK, C. J.; JAMES, C. M. Do Banks Provide Financial Slack? Journal of Finance, v. 57, n. 3, p. 1383-1419, 2002.

HUANG, G.; SONG, F. M. The determinants of capital structure: Evidence from China.

China Economic Review, v. 17, n. 1, p. 14-36, 2006.

JENSEN, M. C. .; MECKLING, W. H. Theory of the Firm : Managerial Behavior, Agency Costs and Ownership Structure. Journal of Financial Economics, v. 3, p. 305-360, 1976. JO, H.; KIM, H.; PARK, K. Corporate Environmental Responsibility and Firm Performance in the Financial Services Sector. Journal of Business Ethics, v. 131, n. 2, p. 257-284, 2014. KESTER, W. C. Management in Japan Capital Ownership Structure : United and Japanese Manufacturing Corporations. Financial Management, v. 15, n. 1, p. 5-16, 1986.

KHAN, M. I. Capital Structure , Equity Ownership and Firm Performance : Evidence from India. Social Science Research Network, p. 1-14, 2012.

LARA, José Edson; MESQUITA, José Marcos Carvalho. Estrutura de Capital e Rentabilidade: análise do desempenho de empresas brasileiras no período pós Plano Real. Contabilidade Vista \& Revista, v. 19, n. 2, 2008.

LAUTERBACH, B.; VANINSKY, A. Ownership Structure and Firm Performance : Evidence 
from Israel. Journal of Management, p. 189-201, 1999.

LELAND, Hayne E.; TOFT, Klaus Bjerre. Optimal capital structure, endogenous bankruptcy, and the term structure of credit spreads. The Journal of Finance, v. 51, n. 3, p. 987-1019, 1996.

MACHADO, Luiz Kennedy Cruz et al. A relevância da estrutura de capital no desempenho das firmas: uma análise multivariada das empresas brasileiras de capital aberto. Revista de Educação e Pesquisa em Contabilidade, v. 9, n. 4, 2015.

MAJUMDAR, S. K.; CHHIBBER, P. Capital structure and performance : Evidence from a transition economy on an aspect of corporate governance. Public Choice, v. 98, n. 3, p. 287$305,1999$.

MARGARITIS, D.; PSILLAKI, M. Capital structure, equity ownership and firm performance. Journal of Banking and Finance, v. 34, n. 3, p. 621-632, 2010.

MARSH, P. R. The Choice between Debt and Equity: An Empirical Study. Journal of Finance, v. 37, n. 1, p. 121-144, 1982.

MICHAELAS, N.; CHITTENDEN, F.; POUTZIOURIS, P. Financial policy and capital structure choice in UK SMEs: evidence from company panel data. Small Business Economics, v. 12, n. 2, p. 113-130, 1999.

MODIGLIANI, F.; MILLER, M. The Cost of Capital, Corporation Finance and the Theory of Investment. The American Economic Review, v. 45, n. 1, p. 1-28, 1958.

PAMPLONA, Edgar; MAGRO, Cristian Baú Dal; SILVA, Tarcísio Pedro da. A ESTRUTURA DE CAPITAL E O DESEMPENHO ECONÔMICO DE EMPRESAS FAMILIARES DO BRASIL E DE PORTUGAL. IX ANPCONT, 2015

PERRESSIM, William Sbrama; SANTOS, David Ferreira Lopes; LUCENTE, Adriano dos Reis. Estrutura de Capital e Desempenho Financeiro no Setor Bancário Brasileiro Entre 1998 e 2006. XI SEGET, 2014.

PHILLIPS, P. A. P.; SIPAHIOGLU, M. A. S. F. MS. Performance implications of capital structure: evidence from quoted UK organisations with hotel interests. The Service

Industries Journal, v. 24, n. 5, p. 31-51, 2004.

PHUNG, D. N.; MISHRA, A. V. Ownership Structure and Firm Performance : Evidence From Vietnamese Listed Firms. v. 2012, 2015.

PRATHEEPKANTH, P. Capital Structure and Financial Performance : Evidence From Selected Business Companies. Journal International refereed research, v. II, n. April 2011, p. 171-183, 2011. 
RAJAN, R. G.; ZINGALES, L. What do we know about capital structure: some evidence from international data. J. Finance, v. 50, n. 5, p. 1421, 1995.

RODEN, D. M.; LEWELLEN, W. G. Corporate Capital Structure Decisions: Evidence from Leveraged Buyouts. FM: The Journal of the Financial Management Association, v. 24, n. 2, p. 76-87, 1995.

SALEHI, M.; BIGLAR, K. Study of the Relationship between Capital Structure Measures and Performance: Evidence from Iran. International Journal of Business and Management, v. 4, n. 1, p. 97-103, 2009.

SALIM, M.; YADAV, R. Capital Structure and Firm Performance: Evidence from Malaysian Listed Companies. Social and Behavioral Sciences, v. 65, n. ICIBSoS, p. 156-166, 2012. SAN, O. T.; HENG, B. Capital Structure and Corporate Performance of Malaysian Construction Sector. International Journal of Humanities and Social Science, v. 1, n. 2, p. 28-36, 2011.

SIMERLY, R. L. .; LI, M. Environmental Dynamism, Capital Structure and Performance: A Theoretical Integration and an Empirical Test. Strategic Management Journal, v. 21, n. 1, p. 31-49, 2000.

SINGH, M.; FAIRCLOTH, S. The impact of corporate debt on long term investment and firm performance. Applied Economics, v. 37, n. 8, p. 875-883, 2005.

SPIESS, D. K.; AFFLECK-GRAVES, J. The long-run performance of stock returns following debt offerings. Journal of Financial Economics, v. 54, n. 1, p. 45-73, 1999.

TAUB, A. J. Determinants of the Firm's Capital Structure. The Review of Economics and Statistics, v. 57, n. 4, p. 410-416, 1975.

TITMAN, S.; WESSELS, R. The Determinants of Capital Structure Choice. The Journal of Finance, v. 43, n. 1, p. 1-19, 1988.

WAHBA, H. Capital structure, managerial ownership and firm performance: evidence from Egypt. Journal of Management and Governance, v. 18, n. 4, p. 1041-1061, 2014. WALD, J. K. How Firm Characteristics Affect Capital Structure: an International Comparison. The Journal of Financial Research, v. XXII, n. 2, p. 161-187, 1999. WIWATTANAKANTANG, Y. An empirical study on the determinants of the capital structure of Thai firms. Pacific-Basin Finance Journal, v. 7, n. 3-4, p. 371-403, 1999. 